\title{
ANALYSE DES RISQUES DU CYCLE DE VIE : APPLICATION À DEUX FILIĖRES ÉNERGÉTIQUES EN FRANCE
}

\author{
Patrick ROUSSEAUX*, Michel LICHOU** \& Osama ALKOSHAK***
}

\begin{abstract}
Résumé - Comme tout le monde devrait le savoir, le développement durable repose sur trois piliers : l'économie, l'environnement et le social. Généralement, l'aspect "santé \& sécurité » est considéré dans le pilier social. Autre postulat international moins bien connu : les trois piliers du développement durable doivent être évalués sur l'ensemble du cycle de vie des activités humaines (Extraction des ressources $\rightarrow$ Fabrication $\rightarrow$ Utilisation $\rightarrow$ Fin de vie). Les méthodes traditionnelles d'analyse des risques ne prennent pas en compte cette notion de cycle de vie. Une nouvelle méthode A.R.C.V. (Analyse des Risques de Cycle de Vie) a été proposée pour que cette notion essentielle soit intégrée dans l'analyse des risques. L'A.R.C.V. est ici présentée et illustrée par une application à deux filières énergétiques : l'éolien (Eo) et le photovoltaïque (PV).
\end{abstract}

Mots clés : risques ; analyse ; management ; cycle de vie ; éolien ; photovoltaïque.

\section{Introduction}

Selon (ISO 1, 2006), le cycle de vie (C.V.) d'un produit est défini par «l'ensemble des activités associées à un produit ou à un service depuis l'extraction des matières premières jusqu'à l'élimination des déchets ».

Pour lever toute ambigüité, il est important de bien distinguer cette première définition de cycle de vie avec une autre utilisée dans des analyses de risque comme par exemple dans (Greiner, 1972) ou (Clusel, 2012) et qui correspond aux différentes étapes (ou âges ou degrés de maturité) d'une activité (et en particulier d'une entreprise).

\footnotetext{
* Professeur des Universités, Université de Poitiers, I.R.I.A.F. (Institut des Risques Industriels et Assurantiels), 11 rue Archimède, 79000 Niort, patrick.rousseaux@univ-poitiers.fr

* Chargé de mission « Transition énergétique », S.I.E.D.S. (Syndicat Intercommunal d'Énergie des Deux-Sèvres), 14 rue NotreDame, 79028 NIORT Cedex, mlichou@sieds.fr

*** Étudiant en Master « Management des Risques industriels et environnementaux », Université de Poitiers, I.R.I.A.F. (Institut des Risques Industriels et Assurantiels), 11 rue Archimède, 79000 Niort.
} 
La notion de cycle de vie qui relève de la définition de (ISO 1, 2006), n'est pas prise en compte dans la méthodologie classique des analyses de risques. Pour intégrer cette notion fondamentale à la méthodologie d'analyse de risques, nous nous sommes appuyés sur la méthodologie de l'A.C.V. (Analyse du Cycle de Vie) qui permet d'évaluer des impacts environnementaux tout le long du C.V. d'un système. Si le système est un produit, on considère généralement les grandes étapes suivantes : l'extraction des matières premières, la fabrication du produit, son utilisation et son élimination en fin de vie. Pour un procédé, on distingue généralement les quatre étapes suivantes : l'extraction des matières premières, la mise en œuvre du procédé, son fonctionnement et son démantèlement en fin de vie. Quelques adaptations de la méthodologie A.C.V. ont été nécessaires pour élaborer une nouvelle méthode appelée par ses auteurs (Aissani et al., 2011) "Analyse des Risques de Cycle de Vie (A.R.C.V.)». Cette méthodologie d'A.R.C.V. est ainsi calquée sur celle de l'A.C.V.

L'objectif de cet article est de présenter notre démarche, de décrire notre nouvelle méthode A.R.C.V. et d'illustrer nos propos par une inédite application industrielle sur deux filières d'énergie renouvelable en France.

\section{Construction de l'A.R.C.V. à partir de l'A.C.V.}

L'A.C.V. permet l'évaluation environnementale d'un système (un produit, un service ou un procédé) par la qualification et la quantification des flux entrants et sortants et des impacts environnementaux potentiels associés (par exemple, changement climatique, impact sur la santé, épuisement des ressources, ...). Cet outil est normalisé par deux normes (ISO 1 \& 2, 2006). L'A.C.V. s'appuie sur un bilan qualitatif et quantitatif des aspects et des impacts environnementaux potentiels d'un système remplissant une fonction donnée. Les spécificités de l'A.C.V. sont de deux ordres : la pensée «cycle de vie » et la quantification des impacts environnementaux. La pensée " cycle de vie » permet de prendre en compte toutes les étapes $\mathrm{du}$ cycle de vie d'un système allant de l'extraction des matières premières, en passant par l'étape de fabrication, l'étape d'utilisation jusqu'à la fin de vie. Tout au long du cycle de vie, les flux entrants et sortants de l'environnement sont identifiés et quantifiés afin d'identifier et quantifier les impacts environnementaux associés (Rousseaux, 2005).

Outre ces deux spécificités, l'A.C.V. est reconnue comme un outil holistique au regard non seulement de la prise en compte de toutes les étapes du cycle de vie mais également au regard de l'exhaustivité des impacts évalués. Cette exhaustivité engendre cependant des limites au regard d'une part de la disponibilité et de la qualité des données à recueillir et d'autre part de la mauvaise connaissance des mécanismes d'impacts entraînant une incertitude des résultats.

Le principal point fort de l'A.C.V. est cette vision de cycle de vie. Cette démarche globale et systématique rend possible l'identification des déplacements de charges environnementales entre les différentes étapes du cycle de vie, en d'autres termes dans le temps et l'espace. Il s'agit de la notion de transfert de pollution. Ces transferts de pollution peuvent apparaître lors d'une tentative d'amélioration d'un système en agissant sur une étape du cycle de vie. L'amélioration d'une étape peut entraîner des conséquences néfastes sur une autre, ailleurs ou à un autre moment. 
Compte tenu des points forts de l'A.C.V. et de sa normalisation par l'ISO, nous nous sommes inspirés de l'A.C.V. pour élaborer l'A.R.C.V.

$\mathrm{Au}$ regard du tableau 1, la méthodologie A.C.V. est théoriquement adaptable à l'A.R.C.V. Chacune des quatre étapes de l'A.C.V. trouve son pendant dans l'A.R.C.V.

\begin{tabular}{|l|l|l|}
\hline $\begin{array}{l}\text { Étapes de l'A.C.V. } \\
\text { (ISO 1 \& 2, 2006) }\end{array}$ & $\begin{array}{l}\text { Contenu des étapes de } \\
\text { l'A.C.V. }\end{array}$ & $\begin{array}{l}\text { Étapes de l'A.R.C.V. et leurs } \\
\text { contenus }\end{array}$ \\
\hline $\begin{array}{l}\text { 1- Définition des objectifs et } \\
\text { du champ d'étude }\end{array}$ & $\begin{array}{l}\text { Objectif, hypothèses, fonction et frontières du système, unité } \\
\text { fonctionnelle }\end{array}$ \\
\hline 2- Inventaire & Bilans matière-énergie & $\begin{array}{l}\text { Identification des situations } \\
\text { dangereuses }\end{array}$ \\
\hline 3- Évaluation des impacts & $\begin{array}{l}\text { Quantification des impacts } \\
\text { environnementaux }\end{array}$ & Évaluation du niveau de risque \\
\hline 3-1 Classification & $\begin{array}{l}\text { Identification des impacts du } \\
\text { système par rapport aux flux } \\
\text { inventoriés }\end{array}$ & $\begin{array}{l}\text { Identification des différentes } \\
\text { typologies d'accident pour chaque } \\
\text { situation dangereuse }\end{array}$ \\
\hline 3-2 Caractérisation & $\begin{array}{l}\text { Estimation des impacts } \\
\text { environnementaux }\end{array}$ & Estimation des risques \\
\hline 3-3 Évaluation & $\begin{array}{l}\text { Normalisation des résultats } \\
\text { et/ou agrégation des impacts }\end{array}$ & $\begin{array}{l}\text { Évaluation des classes de risque et } \\
\text { /ou agrégation des risques }\end{array}$ \\
\hline 4- Interprétation des résultats & $\begin{array}{l}\text { Analyse des résultats obtenus et propositions des pistes } \\
\text { d'améliorations }\end{array}$ \\
\hline
\end{tabular}

Tableau 1 : Adaptation de l'A.C.V. à l'A.R.C.V. (Aissani et al., 2011).

Les différences entre ces deux méthodologies se situent aux deux étapes clés de l'A.C.V. : l'inventaire et l'évaluation des impacts / niveau de risque.

L'inventaire permet de recueillir des données dont la nature est différente entre les deux outils. Pour l'A.C.V. les données à recueillir sont des flux de matières et d'énergie, ce sont donc des données qualitatives et quantitatives. Pour l'A.R.C.V. les données à recueillir sont uniquement qualitatives puisqu'il s'agit d'inventorier les situations dangereuses. L'étape d'évaluation des impacts/niveau de risque se compose de trois phases (la classification, la caractérisation et l'évaluation) et permet de réaliser une conversion des données d'inventaire en résultats d'impacts/niveau de risque. Pour l'A.C.V. cette conversion des données d'inventaire est réalisée par un calcul à l'aide d'indicateurs de catégories d'impacts. Pour l'A.R.C.V. cette conversion est réalisée de manière qualitative en cotant et en hiérarchisant les risques. Néanmoins, l'objectif de la phase de classification est identique aux deux outils car elle permet de faire le lien entre les données de l'inventaire et les impacts/accidents à évaluer.

Tout comme les flux identifiés par l'A.C.V. qui peuvent contribuer à différentes catégories d'impacts, les situations dangereuses peuvent engendrer différentes typologies d'accidents. Cependant, l'exhaustivité dans l'inventaire de l'A.C.V. ne peut pas être raisonnablement préconisée pour celui de l'A.R.C.V. En effet, les différentes typologies de risques sont bien trop nombreuses comparées au nombre de catégories d'impacts environnementaux évalués par l'A.C.V. Par ailleurs, l'exhaustivité des typologies de risques ne semble pas pertinente dans une démarche d'analyse globale des risques. Dès lors, des limites et des hypothèses ont été posées dans l'A.R.C.V. afin de rendre l'étude faisable et pertinente au regard des objectifs et de l'applicabilité des résultats attendus. 
Nous n'avons pas été les seuls à avoir eu l'idée de proposer une méthode intégrant la notion de cycle de vie dans l'analyse des risques. Une équipe chinoise a proposé, à la même époque, la même démarche avec les mêmes motivations et en utilisant le même terme L.C.R.A. pour «Life Cycle Risk Assessment» (Hsiu-ching, 2011). Nous présentons dans la suite la méthode française en l'appliquant à deux filières énergétiques, l'éolien (Eo) et le photovoltaïque (PV) et nous indiquerons ensuite la différence avec la méthode chinoise. Enfin, une comparaison entre l'A.R.C.V. et les méthodes traditionnelles d'analyse des risques sera réalisée.

\section{Déroulement de la méthode A.R.C.V. et application aux deux filières énergétiques photovoltaïque et éolienne}

\subsection{Définition des objectifs et du champ de l'étude}

Cette première étape définit les objectifs de l'étude, les cycles de vie étudiés, les frontières du système et les hypothèses associées. Compte tenu de la complexité du C.V., l'A.R.C.V. telle que présentée ici ne considère que les risques d'accident mortel. Il faut également définir l'unité fonctionnelle qui représente une mesure de la performance délivrée par les cycles de vie étudiés. Toute comparaison de cycles de vie doit être réalisée sur la même performance (ou service rendu). Comme pour l'A.C.V., il est important de vérifier que l'unité fonctionnelle est bien en adéquation avec l'objectif de l'étude.

Le commanditaire de l'application de l'A.R.C.V. aux filières Eo et PV est le Syndicat Intercommunal d'Énergie des Deux-Sèvres. Les objectifs sont de trois ordres :

- comparer deux filières de production d'électricité d'origine renouvelable sur d'autres critères que les seuls aspects économiques et l'acceptabilité sociale des projets ;

- élargir l'évaluation des impacts environnementaux et analyser les risques sur l'ensemble du cycle de vie pour les filières Eo et PV qui offrent le même service (production d'électricité) mais avec des caractéristiques très différentes (taille unitaire des installations, mode de raccordement au réseau, intermittence de la production, lieu de fabrication des composants, recyclage en fin de vie, etc.) ;

- identifier les points faibles des deux filières afin de les améliorer.

Les cycles de vie des filières énergétiques étudiées sont présentés dans le tableau 2.

L'étape fabrication d'Eo n'a pas été retenue faute de données fiables. On a fait l'hypothèse que tous les moyens de prévention et de protection sont mis en place et respectés.

L'unité fonctionnelle choisie est la production de 25.000 MWh d'électricité par an, correspondant à la production annuelle moyenne d'un parc éolien de $12 \mathrm{MW}$, raccordé au réseau HTA (moyenne tension). Pour le photovoltaïque en toiture, ceci correspond environ à $25.000 \mathrm{kWc}$ de panneaux photovoltaïques soit près de $165.000 \mathrm{~m}^{2}$, correspondant à plusieurs centaines voire milliers d'installations raccordées au réseau basse tension. 


\begin{tabular}{|l|l|l|}
\hline Étape de cycle de vie & Filière éolienne & Filière photovoltaïque \\
\hline $\begin{array}{l}\text { Extraction et } \\
\text { préparation des } \\
\text { matières premières }\end{array}$ & $\begin{array}{l}\text { Extraction et préparation de: } \\
\text { aluminium, cuivre, acier, fibre } \\
\text { de verres, polyester, huile, } \\
\text { béton }\end{array}$ & $\begin{array}{l}\text { Extraction et préparation de: aluminium, } \\
\text { cuivre, silicium, verre } \\
\text { Extraction et Préparation des produits } \\
\text { chimiques }: \text { HF, NaOH, HNO3, POCl3, NH3, } \\
\text { CH4, HCl, CH3OH }\end{array}$ \\
\hline $\begin{array}{l}\text { Fabrication des } \\
\text { somposants de } \\
\text { système }\end{array}$ & $\begin{array}{l}\text { Fabrication de }: \text { mât, nacelle, } \\
\text { pales, équipements } \\
\text { électriques }\end{array}$ & $\begin{array}{l}\text { Décapage des plaques } \rightarrow \text { texturation } \rightarrow \\
\text { dopage } \rightarrow \text { bords de plaque } \rightarrow \text { couche anti- } \\
\text { reflet } \rightarrow \text { métallisation } \rightarrow \text { raccordement des } \\
\text { cellules } \rightarrow \text { encapsulation } \rightarrow \text { encadrement et } \\
\text { connexion. } \\
\text { Fabrication des équipements électriques }\end{array}$ \\
\hline $\begin{array}{l}\text { Construction } \\
\text { réseau }\end{array}$ & $\begin{array}{l}\text { Préparation du site } \rightarrow \\
\text { préparation des fondations } \rightarrow \\
\text { montage des composants } \rightarrow \\
\text { installation électrique }\end{array}$ & $\begin{array}{l}\text { Préparation de la toiture } \rightarrow \text { montage des } \\
\text { modules } \rightarrow \text { installation électrique }\end{array}$ \\
\hline Fonctionnement & $\begin{array}{l}\text { Raccordement au réseau } \\
\text { moyenne tension }\end{array}$ & Production d'électricité \\
\hline Démantèlement & Démantèlement des composants \\
\hline $\begin{array}{l}\text { Élimination des } \\
\text { déchets }\end{array}$ & Recyclage, mise en décharge, incinération \\
\hline Transports & Camion, bateau, voie ferrée, voie maritime \\
\hline
\end{tabular}

Tableau 2 : Étapes du cycle de vie des filières éolienne et photovoltaïque (Alkoshak, 2011).

\subsection{Inventaire des situations dangereuses}

Cette étape consiste en la détermination des situations dangereuses pour chaque étape du cycle de vie. Les résultats de cet inventaire des situations dangereuses par étape et par activité humaine sont présentés dans les lignes a, b \& c du tableau 3.

\begin{tabular}{|l|l|l|l|}
\hline $\mathbf{a}$ & Étape & Extraction et préparation des matières premières \\
\hline $\mathbf{b}$ & Sous étape & Extraction et préparation de la bauxite \\
\hline $\mathbf{c}$ & Situation dangereuse & Utilisation des matières explosives \\
\hline $\mathbf{d}$ & Accident & Explosion \\
\hline & \multirow{2}{*}{$\begin{array}{l}\text { Moyens de } \\
\text { maitrise }\end{array}$} & Protection & $\begin{array}{l}\text { Etablir un plan d'intervention } \\
\text { Port d'équipement de protection individuelle }\end{array}$ \\
\cline { 2 - 4 } & Prévention & $\begin{array}{l}\text { Formation du personnel } \\
\text { Respecter les consignes de sécurité }\end{array}$ \\
\hline $\mathbf{f}$ & Réglementation & ATEX (ATmosphères EXplosibles) \\
\hline $\mathbf{g}$ & P & 1 \\
\hline $\mathbf{h}$ & E & 1 \\
\hline
\end{tabular}

Tableau 3 : Extrait du tableau d'inventaire des situations dangereuses et des accidents associés à la filière éolienne (Alkoshak, 2011).

Pour identifier les situations dangereuses, deux méthodes complémentaires sont utilisées : 
- une recherche «a priori»: il s'agit d'identifier les situations susceptibles d'engendrer un accident. Afin de réduire les erreurs et les omissions, l'A.R.C.V. préconise d'utiliser la liste des sources de danger de la méthode MOSAR (Perilhon, 2003). Cette liste considérée comme exhaustive, retient 9 catégories de sources de danger avec 46 sous-catégories ;

- une recherche "a posteriori » : il s'agit d'identifier les situations ayant déjà engendré des accidents en utilisant la base de données du BARPI (disponible en ligne sur le site http: / / www.aria.developpement-durable.gouv.fr/) ou d'autres sources bibliographiques.

Dans notre application, les filières Eo et PV comptabilisent respectivement 253 et 306 situations dangereuses.

\section{3 Évaluation du niveau de risque}

\subsubsection{Classification}

Cette étape de classification permet de faire le lien entre les situations dangereuses et les accidents. Pour chaque situation dangereuse, les différents accidents possibles sont identifiés (ligne d du tableau 3). Pour chaque accident, les moyens de maîtrise en termes de prévention et de protection prévus par la loi sont inventoriés (ligne e du tableau 3) ainsi que les textes réglementaires associés (ligne $\mathrm{f} d u$ tableau 3). Chaque situation dangereuse peut donner lieu à un ou plusieurs accidents.

Dans notre application, les différentes typologies d'accidents que nous pouvons rencontrer, le plus souvent, sont : l'incendie, les traumatismes physiques, l'explosion, l'accident routier, l'inhalation et le choc électrique. Nous avons répertorié pour les filières Eo et PV respectivement 315 et 339 accidents potentiels. Pour la filière Eo, deux typologies se dégagent : le traumatisme physique $(31 \%)$ et l'explosion $(28 \%)$. Pour la filière PV, trois typologies se dégagent : l'explosion (29\%), le traumatisme physique (23\%), et l'inhalation $(18 \%)$.

\subsubsection{Caractérisation}

La prise en compte de la globalité du cycle de vie introduit la prise en compte d'un nombre de données trop important. La quantification ou caractérisation des risques rencontrés en termes de probabilité d'occurrence et de gravité, apparaît alors ardue. Pour s'affranchir de ce problème, l'A.R.C.V. réalise une évaluation qualitative des risques en les hiérarchisant.

Le terme de probabilité d'occurrence est évalué grâce à la détermination du nombre de défaillances et de leurs relations pouvant mener à l'événement létal. Le terme de gravité détermine la nature des dommages provoqués. Leur nature n'étant dans l'A.R.C.V. que la mort, il semble difficile de déterminer différents niveaux. L'A.R.C.V. évalue donc leur étendue dans l'espace.

L'A.R.C.V. propose alors deux échelles de cotation :

- l'échelle de détermination de la probabilité d'occurrence $(P)$;

- l'échelle de détermination d'étendue des dommages (E). 
L'échelle de détermination de P se compose des trois niveaux suivants :

- Niveau 1 (le plus faible): la concomitance d'au moins deux défaillances indépendantes est nécessaire pour déclencher l'événement mortel ;

- Niveau 2 : une défaillance spécifique (et seulement une seule) peut déclencher l'événement mortel ;

- Niveau 3 (le plus fort): plusieurs défaillances indépendantes peuvent déclencher l'événement mortel.

Exemples d'application :

- l'apparition d'un feu est classée en niveau 1 car il faut réunir trois conditions concomitantes: la présence du combustible, du comburant et de la source d'ignition ;

- un accident de voiture est classé en niveau 3 car de nombreuses conditions indépendantes peuvent mener à l'accident: présence d'un autre véhicule, défaillance mécanique, fatigue, ivresse, inattention ...

L'échelle de détermination de $\mathrm{E}$ se compose de deux niveaux :

- Niveau 1: dommages localisés au lieu de l'accident ou au site industriel ne touchant que les travailleurs (ou les personnes présentes sur le site) ;

- Niveau 2: dommages susceptibles de s'étendre à l'extérieur du site en atteignant le public.

Exemples d'application :

- un accident provoqué par la manipulation d'un appareil ou d'une machine est classé en niveau 1 car les conséquences ne touchent, la plupart du temps, que l'opérateur ;

- une explosion est classée en niveau 2 car elle engendre un souffle et une propulsion de débris pouvant atteindre des personnes extérieures au site.

- La différence entre ces deux niveaux implique la prise en compte de la probabilité d'extension des dommages à l'extérieur du site. Cette probabilité ne peut pas être exprimée de manière explicite au regard du nombre insuffisant de données. Il s'agit seulement du potentiel implicite d'extension des dommages à l'extérieur du site. Les accidents de type incendie ou explosion sont des accidents susceptibles de s'étendre à l'extérieur du site et sont classés systématiquement en niveau 2. L'accident de la route est également classé en niveau 2 car il présente une forte probabilité d'extension des dommages à une personne du public présente sur la route (dans un autre véhicule impliqué ou sur la voie publique en tant que piéton). En outre, il est important de noter que le passage du niveau 1 au niveau 2 pourrait sous-entendre l'augmentation du nombre de morts et cette échelle serait donc une classification du niveau des dommages par la quantification du nombre de morts. Or ce n'est pas le but de cette échelle. D'une part, l'augmentation du nombre de morts dépend de nombreux paramètres tels que le nombre de personnes présentes sur le site ou à 
proximité, au jour et à l'heure de l'accident, etc. D'autre part, dans une démarche d'acceptation sociale, il semble plus pertinent de s'intéresser au type de cible touchée (travailleur ou public). Par ailleurs, au regard de la disponibilité des données, il apparaît difficile de quantifier le nombre de morts par type de cible.

Pour chaque accident, le niveau de $\mathrm{P}$ (probabilité d'occurrence) et le niveau de $\mathrm{E}$ (étendue des dommages) sont respectivement mentionnés dans les lignes $\mathrm{g} \& \mathrm{~h}$ du tableau 3.

Il est important de noter que les moyens de maîtrise en termes de prévention et de protection ne sont présents qu'à titre informatif. Ils n'ont pas été utilisés lors de la caractérisation.

Afin d'exploiter les nombreux résultats de la cotation, l'A.R.C.V. établit un système de classement des différents scores obtenus en différenciant trois classes de risque selon le score (Niveau de P, niveau de E). Ainsi, comme présenté dans la figure suivante, la classe 1 ne comporte qu'un type de score, la classe 2 deux types et la classe 3 trois types:

G

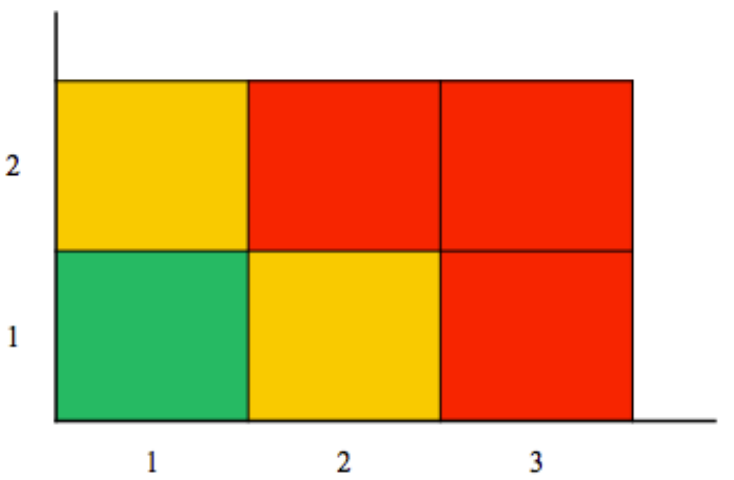

Classe 1

Classe 2

Classe 3

Figure 1 : Les différentes classes des risques de la méthode A.R.C.V. (Aissani et al., 2011).

L'A.R.C.V. se contente d'un nombre maximum de trois classes afin de simplifier l'analyse. Un nombre plus important de classes n'est pas pertinent par rapport au degré de finesse de l'outil.

Dans notre application, la filière Eo comptabilise 10,8\% de classe $1 ; 44,4 \%$ de classe 2 et $44,8 \%$ de classe 3 alors que la filière PV révèle $10 \%$ de classe $1 ; 51,5 \%$ de classe 2 et $38,5 \%$ de classe 3.

Afin de comparer plus aisément les cycles de vie, l'A.R.C.V. prévoit d'élaborer des graphiques appelés "risqueprofils", comparables aux "écoprofils » réalisés en A.C.V. Ces graphiques permettent de visualiser le nombre d'accidents par classe pour chaque grande étape des cycles de vie. Les risqueprofils de notre application sont présentés dans la figure 2.

En comparant les graphiques, on constate que :

- Seules les phases préparation des matières premières et transport comptent des accidents de classe 1 pour les deux filières ; 
Analyse des risques du cycle de vie :
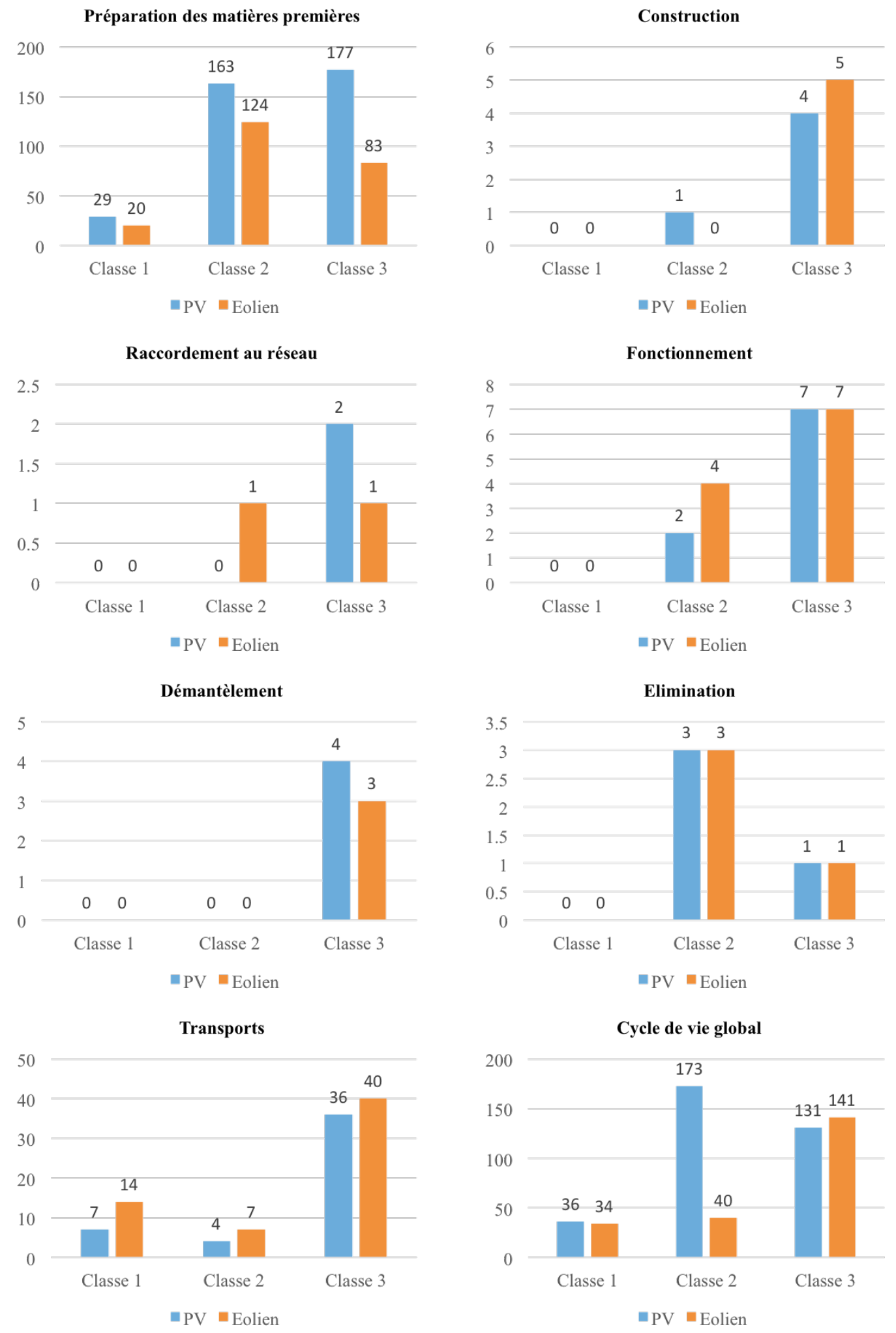

Figure 2 : Risqueprofils : nombre d'accidents possibles par classe de risque des étapes du cycle de vie de l'Éolien et du Photovoltaïque (Alkoshak, 2011). 
- Pour la filière PV, la phase la plus dangereuse semble être l'extraction des matières premières suivie par la phase de transport. On remarque la même chose pour la filière Eo ;

- Pour la phase préparation des matières premières, la filière PV semble être plus dangereuse que la filière Eo en comptabilisant le plus grand nombre d'accidents possibles pour toutes les classes et également pour le nombre total d'accidents possibles ;

- Au contraire, la phase de transport de la filière Eo montre le plus grand nombre d'accidents pour toutes les classes et également pour le nombre total d'accidents. Ceci est compréhensible puisque l'Eo nécessite des convois exceptionnels ;

- Pour la phase de construction, les deux filières montrent le même nombre d'accidents. Cependant la filière Eo comporte 5 accidents en classe 3 alors que la filière PV comporte 4 accidents en classe 3 et 1 accident en classe 2 . On est pourtant sur un nombre limité de grands chantiers pour l'Eo alors que cela correspond à une multitude de petits chantiers pour le PV ;

- Pour la phase de fonctionnement, la filière Eo montre le plus grand nombre d'accidents en classe 2 et le même nombre en classe 3. Cependant elle a globalement le plus grand nombre d'accidents ;

- Pour la phase de démantèlement, les deux filières comportent presque le même nombre d'accidents en classe 3, 3 pour la filière Eo et 4 pour la filière PV ;

- Pour la phase d'élimination, les deux filières sont similaires par rapport au nombre total d'accidents et par rapport au classement.

- Pour la phase de raccordement au réseau, les deux filières ont le même nombre d'accidents en général. Cependant, la filière Eo comporte 1 accident en classe 2 et 1 accident en classe 3 tandis que la filière PV comporte 2 accidents en classe 3 ;

- Globalement, la filière PV comporte le plus grand nombre d'accidents. En considérant toutes les phases étudiées, on remarque presque le même nombre d'accident en classe 1 et classe 3 mais une différence considérable en classe 2.

\subsection{3 Évaluation}

Comme pour l'A.C.V., cette sous-étape méthodologique est éventuellement réalisée pour faciliter la comparaison des cycles de vie à partir d'une méthode d'analyse multicritère. L'A.R.C.V. propose d'agréger les résultats par une moyenne arithmétique pondérée pour obtenir in fine un score unique par cycle de vie étudié. Un coefficient de pondération est attribué à chaque classe de risque : un coefficient 1 pour les accidents de classe 1 , un coefficient 2 pour les accidents de classe 2 et un coefficient 3 pour les accidents de classe 3 . Le score global est donné par la formule suivante :

$$
\text { Score global }=((\mathrm{n} 1 \times 1)+(\mathrm{n} 2 \times 2)+(\mathrm{n} 3 \times 3)) /(\mathrm{n} 1+\mathrm{n} 2+\mathrm{n} 3)
$$

où ni représente le nombre d'accidents possibles par classe i. 
Pour chacune des filières et pour chaque phase du cycle de vie, on obtient des scores en termes de niveau de risque, ces scores sont compris entre les valeurs 1 et 3 , où 1 représente le niveau de risque le plus faible alors que 3 représente le niveau de risque le plus fort. Comme cette pondération est forcément subjective, il est nécessaire, à l'instar de A.C.V., de réaliser une étude de sensibilité sur cette pondération.

\begin{tabular}{|l|l|l|l|}
\hline Description & Score PV & Score Eo & Ecart $\%$ \\
\hline Préparation des matières premières & 2,2 & 2,3 & 4 \\
\hline Fabrication & 2,4 & $/$ & $/$ \\
\hline Construction & 2,8 & 3 & 7 \\
\hline Raccordement au réseau & 3 & 2,5 & 17 \\
\hline Fonctionnement & 2,8 & 2,6 & 5 \\
\hline Démantèlement & 3 & 2,8 & 7 \\
\hline Élimination des déchets & 2,2 & 2,2 & 0 \\
\hline Transports & 2,6 & 2,4 & 7 \\
\hline
\end{tabular}

Tableau 4 : Scores globaux des deux filières pour chaque phase du cycle de vie (Alkoshak, 2011).

\subsection{Interprétation des résultats}

Cette dernière étape de l'A.R.C.V. permet de vérifier la pertinence des résultats par rapport aux finalités et objectifs de l'étude. Différents aspects doivent être passés en revue : les typologies d'accidents répertoriés et leur répartition, les scores des deux filières et la significativité des résultats.

\subsubsection{Examen des différentes typologies d'accidents}

L'examen du tableau 5 permet dans un premier temps, de mettre en évidence un nombre différent d'accidents potentiels entre la filière Eo et la filière PV.

\begin{tabular}{|l|l|l|l|l|}
\hline & PV & Eo & \multicolumn{2}{l|}{} \\
\cline { 2 - 5 } & Nombre & $\%$ & Nombre & $\%$ \\
\hline Traumatisme & 76 & 23 & 97 & 31 \\
\hline Accident routier & 34 & 10 & 34 & 11 \\
\hline Incendie & 30 & 9 & 32 & 10 \\
\hline Explosion & 100 & 29 & 90 & 28 \\
\hline Inhalation & 60 & 18 & 31 & 10 \\
\hline Choc électrique & 14 & 4 & 7 & 2 \\
\hline Autre & 25 & 7 & 24 & 8 \\
\hline Total & 339 & 100 & 315 & 100 \\
\hline
\end{tabular}

Tableau 5 : Comparaison des filières Éolienne et Photovoltaïque par typologie d'accidents (Alkoshak, 2011).

Ces deux filières comptabilisent respectivement 315 et 339 accidents potentiels. Au premier abord, une conclusion pourrait s'imposer : la filière présentant le plus grand nombre d'accidents est la filière la plus dangereuse. Cependant, au regard de la disponibilité des données, l'inventaire des situations dangereuses et de leurs accidents associés ne peut pas 
être considéré comme exhaustif, donc il semble pertinent d'examiner les résultats en s'affranchissant du nombre d'accidents. Pour cela, on a calculé la répartition de ces différentes typologies au sein de chaque filière. On a ensuite examiné les différentes typologies d'accidents les plus représentées au sein de chacune des filières. Pour la filière PV trois typologies se dégagent : l'explosion (29\%), le traumatisme physique $(23 \%)$ et l'inhalation $(18 \%)$. Pour la filière Eo deux typologies se dégagent : le traumatisme physique $(31 \%)$ et l'explosion (29\%). L'accident « inhalation » est plus présent dans la filière PV (18\%) que la filière éolienne $(10 \%)$. Ceci peut s'expliquer par le grand nombre de matières premières chimiques utilisées dans la filière PV. Beaucoup de ces produits chimiques sont toxiques et leur production comporte des risques d'inhalation. L'accident «traumatisme physique » est plus présent dans la filière Eo $(31 \%)$ que la filière PV $(23 \%)$. Cela s'explique par le grand nombre de métaux nécessaires pour la fabrication d'une éolienne, qui nécessite une série de procédés accidentogènes (extraction, broyage, affinage, fonte, coulée, refroidissement, etc).

\subsubsection{Scores et classement des phases du cycle de vie et des deux filières}

Au regard des résultats du tableau 4, il est possible d'établir un classement par ordre décroissant des niveaux de risques des différentes phases du cycle de vie pour chacune des filières :

- Filière PV : raccordement au réseau / démantèlement, construction, fonctionnement, transport, fabrication, élimination, préparation des matières premières.

- Filière Eo : construction, démantèlement, fonctionnement, raccordement au réseau, transport, préparation des matières premières, élimination.

Dans un premier temps on a présumé que la phase de préparation des matières premières est la phase la plus dangereuse au sein de la filière PV. Mais à la lumière de ces données, il s'avère que la phase de raccordement au réseau est la plus dangereuse. Ceci peut s'expliquer par le nombre d'accidents global de la phase où on trouve 2 accidents de classe 3 , c'est-à-dire que $100 \%$ d'accidents de cette phase sont de classe 3. Par contre, on trouve dans la phase de préparation de matières premières 227 accidents. Parmi ces accidents, on en compte 83 de classe 3, 124 de classe 2 et 20 de classe 1 . En conséquence, 36,6\% d'accidents de cette phase sont de classe $3,54,6 \%$ de classe 2 et $8,8 \%$ de classe 1 .

Certains scores étant relativement proches, on a calculé les écarts relatifs des valeurs pour une même phase entre les deux filières selon la formule suivante :

((valeur la plus forte - valeur la plus faible) / valeur la plus forte) x 100

Le calcul de ces écarts permet de vérifier si les valeurs sont significativement différentes. Au regard du tableau 4, on remarque que le score le plus important pour la filière Eo est celui obtenu pour la phase de construction. Pour la filière PV les scores supérieurs sont obtenus pour les phases de démantèlement et de raccordement au réseau. La phase de raccordement au réseau de la filière PV est considérablement plus dangereuse que celle de la filière Eo. Ceci peut aussi s'expliquer par le nombre d'installations concernées : 1 seul parc éolien correspond à plusieurs centaines de toitures photovoltaïques pour un productible comparable. 
La figure 3 indique que la filière PV semble légèrement plus dangereuse pour les trois phases : transport, fonctionnement, démantèlement.

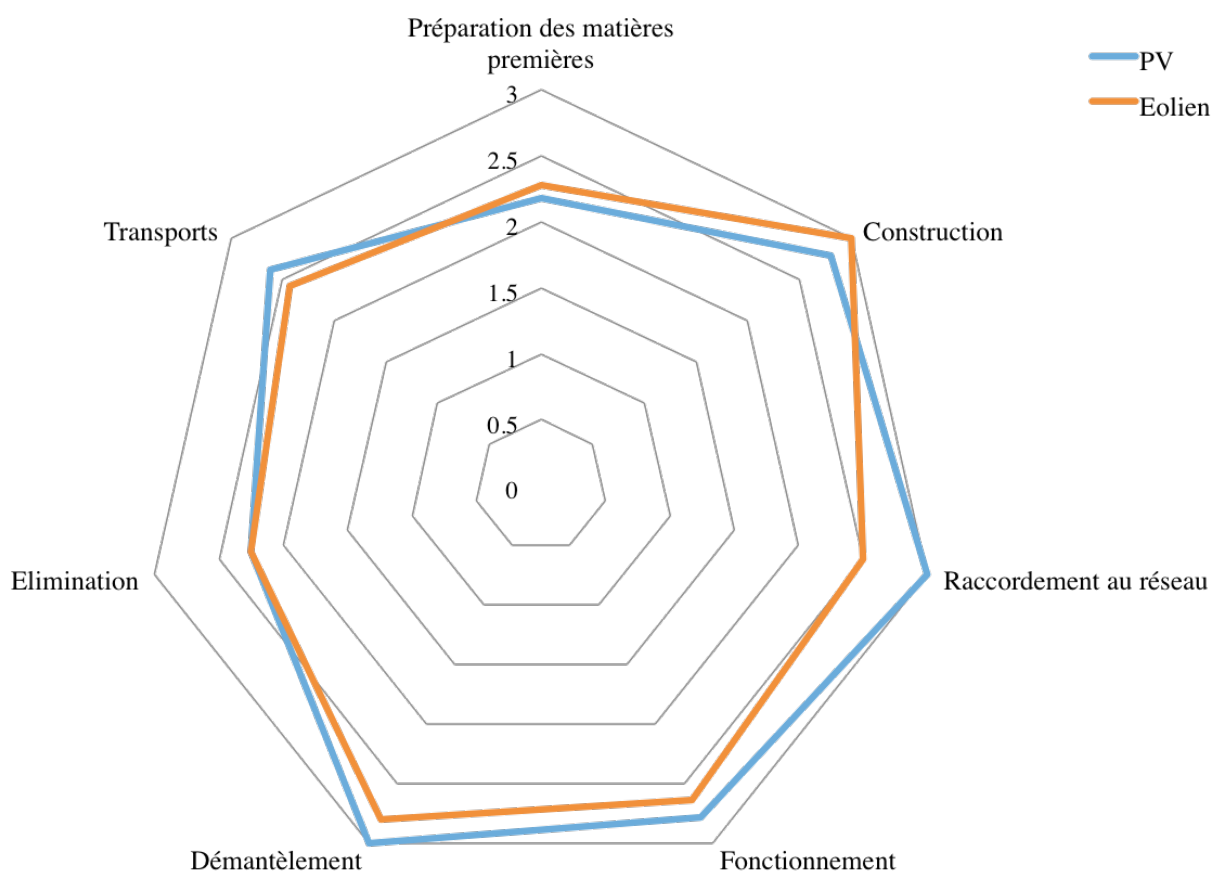

Figure 3 : Visualisation graphique des scores obtenus par phase du cycle de vie des filières Éolien et Photovoltaïque (Alkoshak, 2011).

L'écart est encore plus net pour la phase raccordement au réseau. Par contre, la filière Eo semble légèrement plus dangereuse pour les phases préparation des matières premières et construction. Pour la phase d'élimination, les deux filières sont semblables.

\subsubsection{Significativité des résultats}

L'étude des résultats globaux des deux filières (tableau 4 et figure 3) a mis en évidence de faibles écarts entre les deux filières. Afin de vérifier la significativité des écarts, il est nécessaire de connaitre l'ordre de grandeur des incertitudes. Du fait de l'hétérogénéité des données et de leur faible disponibilité, l'ordre de grandeur des incertitudes n'a pu être déterminé. En effet, nos résultats sont directement liés à l'inventaire des situations dangereuses et à la détermination des niveaux de risque. Pour les deux filières, l'analyse a posteriori pour tous les accidents potentiels de la filière n'a pas été faite car ces 2 filières sont relativement récentes. L'inventaire des situations dangereuses n'est pas exhaustivement identifié et établi. Par ailleurs, la réalisation de la cotation peut également constituer un biais dans la méthode. La probabilité d'occurrence d'un accident est déterminée grâce au recensement des défaillances susceptibles de le provoquer. Ce recensement n'est pas exhaustif et il est donc probable que, comme toutes les défaillances n'ont pas été identifiées, le niveau attribué soit inexact. Ainsi, la différence de niveaux attribués peut engendrer des résultats potentiellement différents et donc des écarts différents. Par ailleurs, l'évaluation du terme étendue des dommages est également soumise à quelques limites. On a systématiquement majoré à un niveau 2 tous les accidents dont les conséquences 
s'étendaient potentiellement hors du site et risquaient d'atteindre le public. Ces accidents sont l'explosion, l'incendie et l'accident routier. La probabilité de l'extension des conséquences de ces accidents n'est pas connue précisément.

Dès lors, il est possible que, selon les circonstances de l'accident, les conséquences soient limitées au site et les accidents concernés ne peuvent donc pas être classés en niveau 2. Ce constat met donc en évidence une majoration du terme étendue des dommages. Dès lors, il apparaît difficile de déterminer un seuil de significativité des écarts calculés.

Le défi de développement de cette méthode serait de passer du qualitatif au quantitatif sans trop alourdir l'analyse. Il s'agirait aussi de prendre en compte d'autres niveaux d'accidents comme des blessures graves n'entraînant pas la mort ou des atteintes environnementales majeures.

En conclusion, les résultats de notre application sont les suivants : pour la filière PV, les étapes les plus problématiques sont le raccordement au réseau et le démantèlement et pour la filière Eo l'étape de construction. Globalement, les écarts relatifs entres les deux filières sont faibles et ne peuvent pas être considérés comme significatifs. Il apparaît donc difficile de trancher en termes de dangerosité entre les deux filières.

Enfin, pour revenir à l'autre proposition méthodologique faite par l'équipe chinoise, la grande et seule différence entre l'A.R.C.V. française et chinoise porte sur le type de risque considéré. Comme nous venons de le voir, la démarche française propose de considérer le risque d'accident mortel quelle que soit la cause alors que la démarche chinoise propose de retenir que le risque toxique. La première démarche est plus exhaustive et la deuxième est plus précise car quantitative.

\section{L'A.R.C.V. et les autres méthodes d'analyse des risques}

Pour mieux positionner la méthode A.R.C.V. par rapport aux autres méthodes d'analyse des risques, nous avons réalisé une analyse multicritère simplifiée en prenant en compte les critères suivants :

- A posteriori / A priori : ce critère indique si la méthode est réalisée à partir d'un évènement survenu ou non ;

- Inductive / Déductive : une méthode inductive est une méthode qui part des causes pour arriver aux effets ; à l'inverse, une méthode déductive part des effets pour arriver aux causes ;

- Vision globale / Vision partielle : la méthode prend en compte l'ensemble du cycle de vie d'un procédé ou d'un produit ou seulement une partie du cycle de vie ;

- Comparaison de différents systèmes : ce critère définit si la méthode étudiée a une vocation intrinsèque à pouvoir comparer différents systèmes ;

- Exhaustivité : ce critère permet d'indiquer si la méthode prend en compte l'ensemble des risques, des causes et des défaillances.

Le tableau 6 permet de savoir si les méthodes étudiées répondent à ces critères. 
De plus, les méthodes d'analyses de risques existantes ont des modes d'évaluation différente du risque.

Certaines utilisent des modèles afin de modéliser la source de danger, d'autres étudient les accidents s'étant déjà produits, d'autres établissent des arbres des causes afin de déterminer des scénarios d'accidents et enfin les dernières n'utilisent que les propriétés physico-chimiques afin d'établir un niveau de risque.

Notre méthode utilise, quand les données sont disponibles, le retour d'expérience $d^{\prime}$ accidents afin d'identifier les situations dangereuses susceptibles de les engendrer. Quand les données n'existent pas ou ne sont pas disponibles, nous utilisons les propriétés physicochimiques des facteurs à risque. En utilisant ces deux démarches, notre méthode tend à être exhaustive et est complémentaire des autres méthodes existantes.

\begin{tabular}{|l|c|c|c|c|c|c|}
\hline & AMDEC1 & HAZOP2 & $\begin{array}{l}\text { Arbre de } \\
\text { défaillances }\end{array}$ & $\begin{array}{l}\text { Arbre de } \\
\text { conséquence }\end{array}$ & MOSAR3 & ARCV \\
\hline A posteriori & - & - & - & + & - & + \\
\hline A priori & + & + & + & - & + & + \\
\hline Inductive & + & + & - & + & + & + \\
\hline Déductive & - & - & + & - & - & - \\
\hline Vision globale & + & - & - & - & - & + \\
\hline $\begin{array}{l}\text { Vision } \\
\text { partielle }\end{array}$ & - & + & + & + & + & - \\
\hline $\begin{array}{l}\text { Comparaison } \\
\text { de systèmes }\end{array}$ & - & - & - & - & - & + \\
\hline Exhaustivité & + & + & + & + & + & - \\
\hline
\end{tabular}

Tableau 6 : Comparaison multicritère des méthodes d'analyse des risques («-» : négatif ; «+» : affirmatif).

Par ailleurs, les méthodes existantes n'étudient généralement qu'une seule cible, les hommes et plus particulièrement les usagers et pas toujours les travailleurs. Dans un contexte d'acceptation sociale, le fait d'axer l'analyse de risques sur cette cible spécifique semble cohérent et judicieux. Nous avons décidé de considérer également les travailleurs car la prise en compte de l'ensemble du cycle de vie des filières énergétiques les met au centre de la plupart des accidents.

Les accidents, dans la plupart des études existantes, peuvent être qualifiés comme «majeurs » ou en d'autres termes entraînant des conséquences graves telles que l'incendie, l'explosion ou l'inhalation avec des dommages dont les conséquences sont irréversibles (la mort). En général, la réglementation et la normalisation mettent l'accent sur les risques «majeurs » en termes d'explosion et/ou d'incendie. 
L'intérêt principal de l'A.R.C.V. est de considérer le C.V. dans une analyse de risques et permet ainsi d'identifier et de quantifier les " transferts de risques ». Par exemple, une étape du C.V. peut s'avérer "sûre " au niveau de ses frontières mais poser des problèmes de sécurité dans les étapes en amont et en aval. On constate que, par rapport aux autres méthodes, l'A.R.C.V. a une vision globale et est capable de comparer les différents systèmes.

Contrairement à des méthodes comme M.O.S.A.R. (Méthode Organisée Systémique d'Analyse des Risques) ou HAZ.OP. (HAZard and OPerability study) par exemple qui analysent dans le détail un système donné, existant ou à créer, l'A.R.C.V. peut être utilisée pour guider des choix généraux de conception : du point de vue des risques évalués sur l'ensemble du cycle de vie, quelle est la meilleure solution ? Elle peut être utilisée alors que la conception de la solution n'en est qu'à ses débuts car elle ne nécessite pas de connaître le détail des installations.

Par exemple dans notre domaine d'application qui est l'énergie, on pourrait utiliser l'A.R.C.V. dans le débat sur la transition énergétique, pour comparer les filières de production d'électricité en analysant le plus objectivement possible les impacts environnementaux et les risques du "berceau à la tombe ", non seulement sur le lieu d'exploitation mais aussi sur les divers lieux concernés par les étapes de fabrication des équipements et leur démantèlement. Vaut-il mieux brûler du charbon, avec un impact certain sur l'environnement et un risque pour l'homme peu grave mais fréquent (dans les mines surtout) ou utiliser du nucléaire à l'impact moindre mais au risque d'accident majeur d'une gravité plus élevée mais d'une probabilité heureusement plus faible ? Vers quelles filières d'énergies renouvelables doit-on orienter les financements publics ? Telles sont des questions pour lesquelles l'A.R.C.V. cherche à apporter des réponses scientifiques et objectives au-delà des premières approches souvent subjectives.

En revanche, la méthode A.R.C.V. est chronophage et peu précise et ne peut être exhaustive compte tenu du nombre important de données à collecter. Ceci limite malheureusement son champ d'application. En effet, compte tenu de la mondialisation des échanges économiques, un C.V. pourrait avoir une étape d'extraction des matières premières en Afrique, une étape de fabrication en Asie et une étape de consommation en Europe, d'où une collecte très difficile des données. Afin résoudre ce problème de disponibilité des données dans le domaine de l'AC.V., il a fallu quelques dizaines d'années pour élaborer des banques de données privées et publiques, qui sont aujourd'hui relativement accessibles.

\section{Conclusion}

L'intérêt politique de considérer le C.V. dans une analyse de risques est d'identifier et de quantifier les transferts de risques. Les grandes instances internationales (O.N.U., U.E.) ont adopté cette approche C.V. car elle permet de vérifier le principe de développement durable et ceci pour chaque famille de critères à considérer : économique, social et environnemental.

L'intérêt scientifique de l'A.R.C.V. est de savoir où il faut agir dans une recherche d'amélioration.

L'intérêt managérial de l'A.R.C.V. est de permettre une comparaison des risques de différents systèmes rendant le même service. Elle peut être très utile dans le contexte d'acceptation sociale de technologies considérées comme dangereuses. Cet aspect est 
d'autant plus important qu'une installation considérée comme " sûre » pendant sa phase d'exploitation sur un territoire donné peut être très dangereuse lors de la construction des composants dans un autre pays.

Compte tenu que l'A.R.C.V. évalue les risques d'accident mortel de tout C.V., il parait difficile d'avoir une bonne précision sur les résultats. L'A.R.C.V. doit être considérée comme un outil d'évaluation grossière des risques.

Enfin, l'A.R.C.V. est à ce jour un outil perfectible. La méthodologie proposée soulève quelques questions en termes d'exhaustivité de l'inventaire des accidents possibles. Telle que la méthode est définie à ce jour, seul le risque d'accident mortel est pris en compte. Il serait judicieux de l'étendre à d'autres risques majeurs comme les blessures graves entrainant une incapacité ou à certaines maladies professionnelles fatales ou invalidantes. On peut aussi envisager de prendre en compte les atteintes majeures et irréversibles à l'environnement. Cependant, il ne sera sans doute pas possible d'aller plus bas dans l'échelle des risques : le nombre de données à traiter serait alors beaucoup trop important.

La considération de l'ensemble du C.V. dans une analyse de risques introduit, indubitablement, la question de l'exhaustivité et de la représentativité des données utilisées. Dans notre exemple, l'étude portait sur les systèmes photovoltaïques placés sur toiture mais il est probable que certaines données proviennent $d$ 'autres types d'installations photovoltaïques.

Enfin, il faudrait passer d'une évaluation qualitative à une évaluation quantitative des risques sans trop alourdir l'analyse.

\section{Bibliographie}

Aissani L., Jabouille F., Bourgois J. \& Rousseaux P., (2011), “A new methodology for risk evaluation taking into account the whole life cycle (LCRA): Validation with case study", Process Safety and Environmental Protection, Vol. 90, N4, pp. 295-303.

Alkoshak O., (2011), Contribution à l'analyse des risques du cycle de vie des filières d'énergie électrique des Deux-Sèvres. Mémoire de stage de Master 2 (M.R.I.E.) : Université de Poitiers (I.R.I.A.F.).

Clusel S., (2012), Définition d'une démarche de réduction des vulnérabilités des TPE/PME fondée sur le concept de cycle de vie, Thèses de doctorat : ParisTech, Mines de Paris.

Greiner L., (1972), "Evolution and Revolution as Organizations Grow, Harvard Business Review", Vol. 50, Issue 4, pp. 37-46.

Hsiu-ching S., Hwong-wen M., (2011), "Life cycle risk assessment of bottom ash reuse", J. of Hazardous Materials, 190, pp. 308-316.

ISO1 (2006), Management environnemental - Analyse du cycle de vie - Principes et cadre, ISO 14040.

ISO2 (2006), Management environnemental - Analyse du cycle de vie - Exigences et lignes Directrices, ISO 14044.

Perilhon P., (2003), « MOSAR : Présentation de la méthode », Techniques de l'Ingénieur, SE 4060.

Rousseaux P., (2005), « Analyse du cycle de vie (A.C.V.) », Techniques de l'Ingénieur, G5500 